\title{
LED Chips and Packaging for 120 LPW SSL Component
}

\author{
Final Report
}

$\begin{array}{ll}\text { Period Start Date: } & \text { October 1, 2007 } \\ \text { Period End Date: } & \text { September 30, } 2009 \\ \text { Principal Investigator: } & \text { James Ibbetson } \\ \text { Date Report Issued: } & \text { December 28, } 2009 \\ \text { Award Number: } & \text { DE-FC26-07NT43225 } \\ \text { Project Manager: } & \text { Ryan Egidi }\end{array}$

Cree Santa Barbara Technology Center

340 Storke Road

Goleta CA 93117

(805) 968-9460 


\section{ABSTRACT}

Cree has developed a new, high-efficiency, low-cost, light emitting diode (LED) lamp module that should be capable of replacing standard, halogen, fluorescent and metal halide lamps based on the total cost of ownership. White LEDs are produced by combining one or more saturated color LEDs with a phosphor or other light down-converting media to achieve white broad-band illumination. This two year project addressed LED chip and package efficiency improvements to establish a technology platform suitable for low-cost, high-efficiency commercial luminaires. Novel photonic-crystal LEDs were developed to improve the light extraction efficiency of blue GaN-based LEDs compared to the baseline technology. Improved packaging designs that reduced down-conversion and absorption related light losses, led to a higher overall LED efficiency.

Specifically, blue LEDs were demonstrated with light output nearing $600 \mathrm{~mW}$ and an external quantum efficiency greater than 60 percent (using $1 \mathrm{~mm}^{2}$ chips at an operating current of 350 $\mathrm{mA}$ ). The results were achieved using a novel, production capable photonic-crystal LED fabrication process. These LEDs formed the basis for a multi-chip white lamp module prototype, which provided 510 lumens light output at a correlated color temperature (CCT) of $3875 \mathrm{~K}$ and an operating current of $350 \mathrm{~mA}$ per $1 \mathrm{~mm}^{2}$ chip. The overall conversion efficiency at $4100 \mathrm{~K}$ improved to $\sim 65 \%$. The resulting efficacy is 112 lumens per watt (LPW) - a 33\% improvement over the start of the project. In addition, a proof-of-concept luminaire was demonstrated that provided a flux of 1700 lumens at a 3842 K CCT.

\section{DISCLAIMER}

This report was prepared as an account of work sponsored by an agency of the United States Government. Neither the United States Government nor an agency thereof, nor any of their employees, makes any warranty, express or implied, or assumes any legal liability or responsibility for the accuracy, completeness, or usefulness of any information, apparatus, product, or process disclosed, or represents that its use would not infringe privately owned rights. Reference herein to any specific commercial product, process, or service by trade name, trademark, manufacturer, or otherwise does not necessarily constitute or imply its endorsement, recommendation, or favoring by the United States Government or any agency thereof. The views and opinions expressed herein do not necessarily state or reflect those of the United States Government or any agency thereof. 


\section{$\underline{\text { Table of Contents }}$}

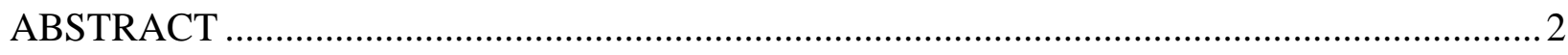

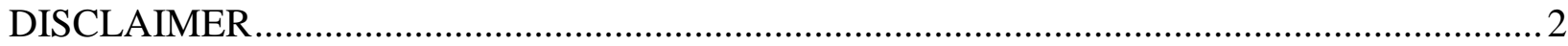

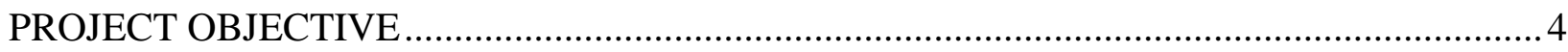

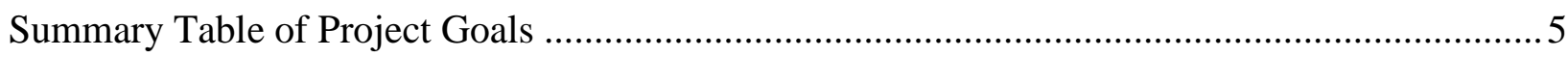

SECTION I - Manufacturable Photonic-Crystal LEDs ................................................ 5

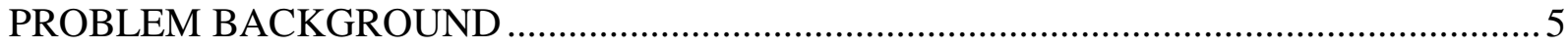

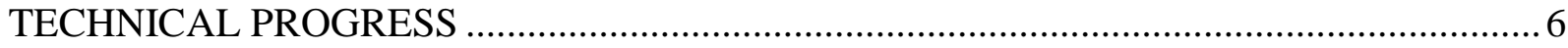

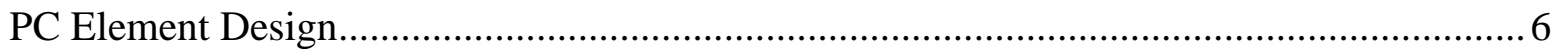

PC Element Materials and Process Development ........................................................ 7

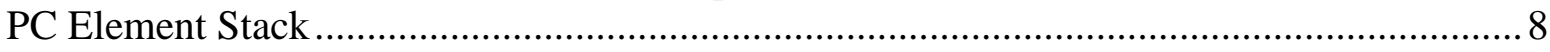

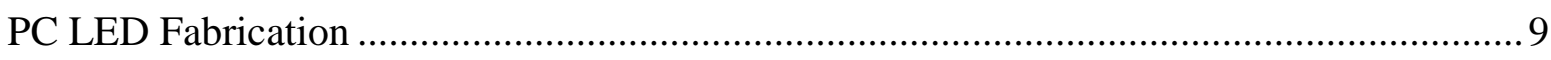

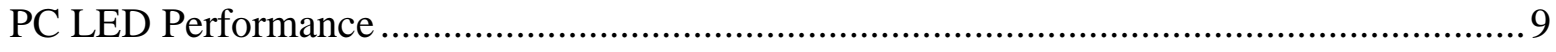

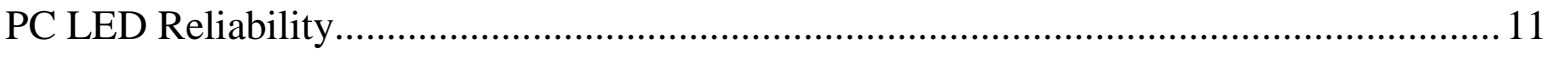

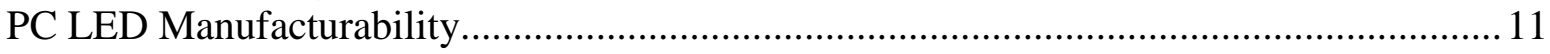

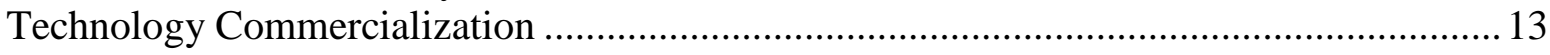

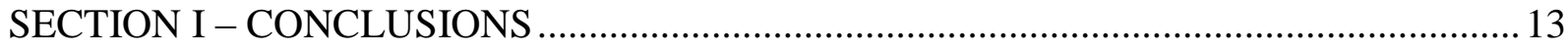

SECTION II - White LED Lamp Module Design ......................................................... 13

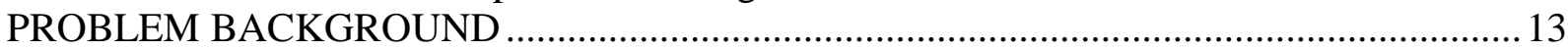

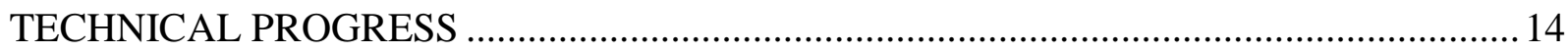

Reflectorless LED Module ..................................................................................... 14

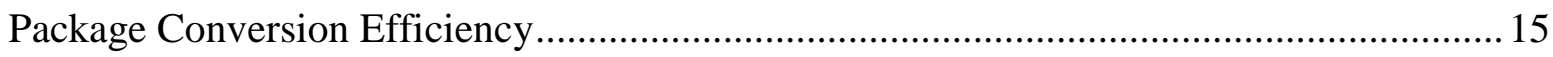

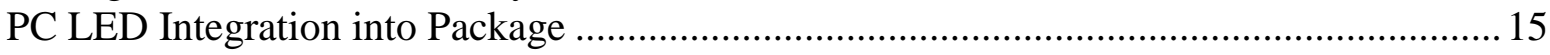

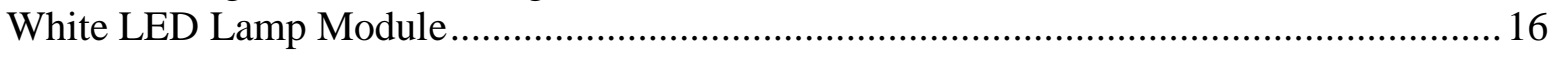

Proof-of-Concept Luminaire Demonstration ............................................................... 17

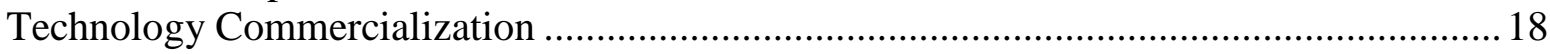

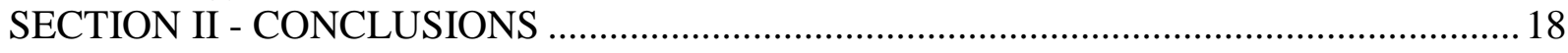

Summary Table of Milestone Performance ..................................................................... 19 


\section{PROJECT OBJECTIVE}

The objective of the project was to develop a novel 120 lumen per watt (LPW) light emitting diode (LED) component that emits white light at a correlated color temperature (CCT) of 4100 $\mathrm{K}$. This component is intended to be the light engine for solid-state luminaires that target the commercial incandescent, halogen, fluorescent and metal halide lamp market. Overall, the two-year project targeted an efficacy gain of $42 \%$ over comparable state-of-the-art product performance at the beginning of the project (Figure 1). To achieve the required efficiency gains, Cree addressed existing optical losses in the areas of chip light extraction efficiency and down-conversion efficiency. The ultimate goal of the program was to deliver to the Department of Energy a white lamp module that produces 500 lumens at an efficacy of 120 LPW and a CCT of $4100 \mathrm{~K}$.

Neutral White Component - Efficiency Breakdown Q4 '07 Status: 95 Im @ 84 Im/W

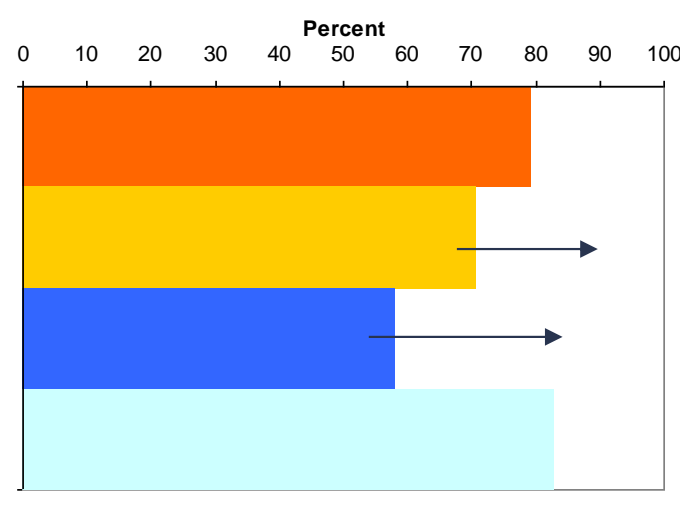

\section{Getting to 120 LPW at 4,100K:}

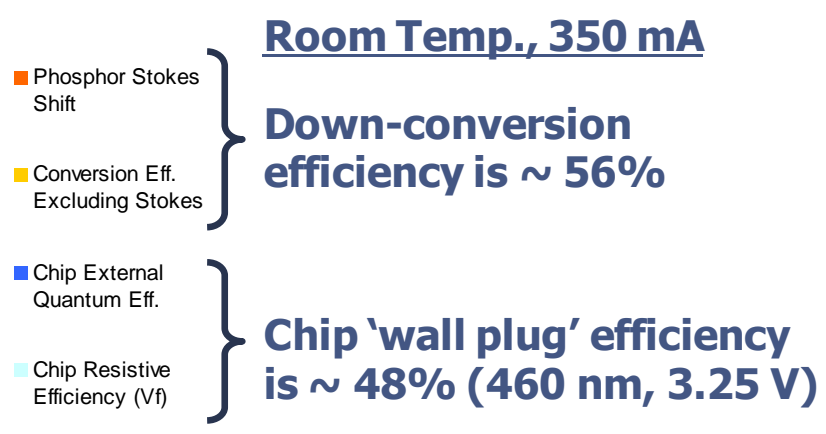

Figure 1: The losses present in the state-of-the-art LED products at the beginning of the project are broken down into two main categories: down conversion efficiency relating to the conversion of blue to white light, and the wall plug efficiency of the blue LED chip. This project targeted a $42 \%$ overall gain in efficacy at $350 \mathrm{~mA}$ by focusing on chip light extraction efficiency and down-conversion efficiency excluding Stokes loss, the current main losses.

To improve chip light extraction, Cree built on its expertise in thin-film InGaN LED technology (as found in its EZBright ${ }^{\circledR}$ product family) by incorporating photonic crystal (PC) technology into the chip design. The goal was to reduce optical losses associated with the mostly random paths of photons in the existing chip via the diffractive effect of PCs located close to the LED active region. Initially, LEDs and test structures were fabricated and characterized in order to qualify materials and processes that make up the PC element, and to provide experimental data to verify and refine optical and electrical models. Later, once proof of concept (i.e. improved light output) was achieved, the project focus shifted to demonstrating the manufacturability of the PC element on 3-inch diameter and larger wafers. Critical parameters, such as PC element thicknesses, were identified and their reproducibility, repeatability and uniformity assessed and improved as needed. By the end of the project Cree aimed to establish a production capable PC-LED fabrication process.

To improve down-conversion efficiency, Cree built on its existing expertise in packaged LEDs (e.g. its XLamp® product family). In one thrust, better package designs and materials were developed with the intent of maximizing first-pass light output. In a second thrust, the phosphor 
particles characteristics were explored to reduce scattering losses. In addition, the location of phosphor particles with respect to the chip was investigated. In order to evaluate progress, prototype LED components were fabricated then characterized for optical performance (mainly brightness) relative to an appropriate baseline process/component. During the project's first year, the phosphor and package development effort used Cree's EZBright chip as the primary (blue) emitter. During the second year, designs were tailored to the newly developed PC LED chip.

This report contains a summary of technical progress made during the two-year project. For the sake of clarity, the report is divided into two main parts according to the two main tasks: Manufacturable Photonic-Crystal LEDs and White LED Module Design. Each part contains background, technical progress, and conclusion sections.

A summary of project milestones by Year and Task is shown in Table 1, below.

\section{Table 1: Summary Table of Project Goals}

\begin{tabular}{|c|l|c|c|}
\hline Task & \multicolumn{1}{|c|}{ Attribute } & Year 1 & Year 2 \\
\hline 2 & Blue PC LED Performance (EQE/Vf) & $55 \%$ & $65 \% / 3.3 \mathrm{~V}$ \\
\hline 3 & 3-Inch PC LED Wafer Uniformity & $\begin{array}{c}<10 \% \text { thickness } \\
\text { variation }\end{array}$ & $\begin{array}{c}+/-10 \% \text { brightness } \\
\text { variation }\end{array}$ \\
\hline 4 & White Module Performance (4100K, 350mA) & $\begin{array}{c}395 \text { lumens } \\
90 \text { LPW }\end{array}$ & $\begin{array}{c}500 \text { lumens } \\
120 \text { LPW }\end{array}$ \\
\hline
\end{tabular}

\section{SECTION I - Manufacturable Photonic-Crystal LEDs}

\section{PROBLEM BACKGROUND}

Cree's EZBright power chip features a proprietary optical design that delivers an optimal lambertian radiation pattern, with low emission losses and high efficiency. In this design (Figure 2), a highly reflective mirror contact is provided on the original LED epiwafer, which is then bonded to a conductive carrier. The substrate is then thinned to reduce bulk absorption, and a textured surface is applied to the remaining material. The underlying principle is that

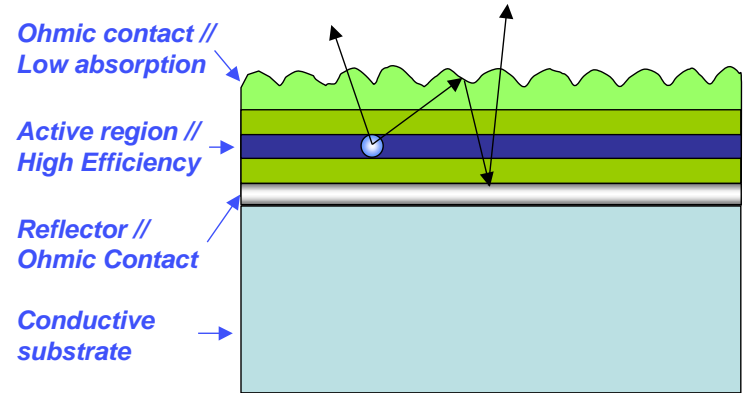

Figure 2: (Left) Schematic of the EZBright LED concept showing its salient features. (Right) Measured efficiency of $465 \mathrm{~nm}$ EZ1000 LED as a function of drive current at the start of the project. Inset: typical device under operation.

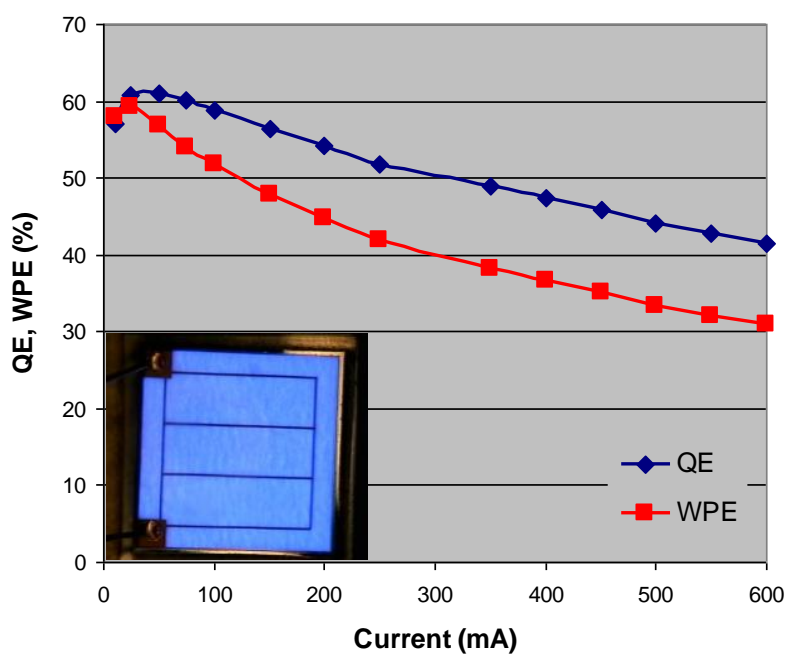


texturing on the surface promotes efficient light extraction by randomizing the paths of any light rays that undergo total internal reflection.

To achieve the overall goal of this program, the task objective was to demonstrate $65 \%$ external quantum efficiency (EQE) from high power blue LEDs. At the start of the project, state-of-theart EZBright LEDs had an EQE of approximately $50 \%$ at $350 \mathrm{~mA}$, as seen in Figure 2. EQE is a product of the internal quantum efficiency (IQE), or how many photons are generated inside the semiconductor per unit of current (essentially materials quality), and the light extraction efficiency, or the fraction of generated photons that escape from the chip. In principle, improvements to the net efficiency can be achieved by improvements to either component. The focus of the effort performed on this project was on increasing the light extraction through the use of photonic crystal technology.

Photonic crystals have been proposed to improve light extraction by diffractively extracting guided modes from the GaN material. ${ }^{1} \quad$ Photonic crystal refers to a repeating structure in an optical medium with the dimensions of the structure being comparable to optical wavelengths. The structure can be one dimensional (1D), 2D or even 3D. As one example shows schematically (Figure 3), a 'leakage' path for the light trapped in the high index layer of the LED can be created using periodic surface patterning (2D PC). In principle, the extraction process can be engineered to be very efficient provided the optical coupling between waveguide modes and "extracted" modes is strong, which is controlled through the design of the waveguide and the photonic crystal. Simulations of PC LEDs show that the total light output can be increased three-fold compared to a conventional LED chip without surface texturing and 1.75 times that of a randomly textured LED. ${ }^{1}$ (It is worth noting though that there was no credible experimental data that Cree was aware of at the start of the project confirming this predicted performance improvement.)

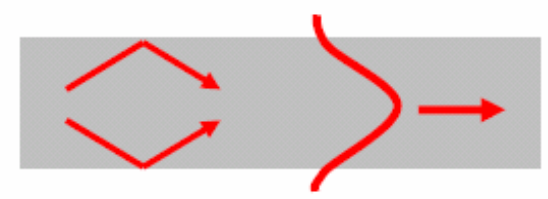

Bouncing light forms a guided mode

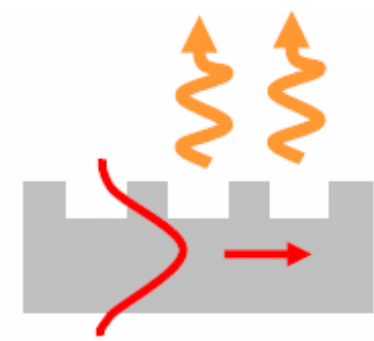

In a periodic structure, the guided mode can be diffracted
Figure 3 Schematic of light extraction from a semiconductor using a 2D photonic crystal.

\section{TECHNICAL PROGRESS}

\section{PC Element Design}

A key first step in the project was the design of the PC-LED itself. Cree limited itself to considering designs that place the PC over the majority of the light emitting area of the LED but do not penetrate into the active region. Other designs for integrating PCs with LED chips have cost or performance drawbacks. For example, incorporating the PC only on a fraction of the chip is not cost effective since the rest of the chip area will not have the brightness enhancements (not fully leveraging the materials costs). Alternatively, etching the PC through

\footnotetext{
${ }^{1}$ M. Zoorob and G. Flinn, LEDs Magazine, p. 21, August 2006.
} 
the active region of the LED decreases the device active area, which in turn increases the current density of the chip and hence decreases its efficiency at the target operating current.

Cree initially investigated two broadly different approaches to incorporating the PC into the LED chip using optical and current injection modeling. One approach places the PC on the pside of the LED, and the other places the PC on the n-side, as shown schematically in Figure 4. Both approaches have some advantages and drawbacks. In the case of the p-side PC, it is generally easier to fabricate the PC element close to the active region, which will allow for stronger coupling of light. A drawback is the known process sensitivity of p-type GaN, which may reduce the efficiency of the chip.

For the n-side PC approach, there will be less material damage problems but the PC will tend to be further from the $\mathrm{QW}$, leading to less optical coupling unless the n-type layers are thinned down. The latter can lead to poorer current spreading and higher operating voltages.
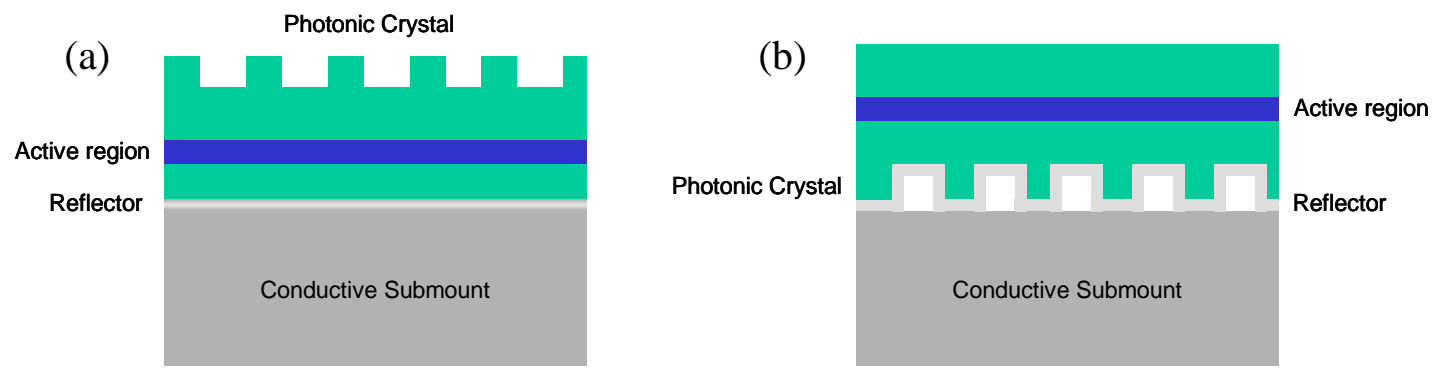

Figure 4: Schematic of PC LED approaches with (a) n-side photonic crystal, (b) p-side photonic crystal.

Based on an extensive trade-off analysis during the first nine months of the project, Cree invented a novel PC-LED design with a p-side PC element that subsequently became the focus of the effort for the remainder of the project. The actual design is proprietary so details cannot be given here but, briefly, it comprises a multi-layer thin film PC element / p-contact, including a transparent conductive oxide (TCO) layer. The selected design has a number of advantages over others that were considered, including relative ease of manufacturing and tolerance to process variations.

Detailed design calculations, which took into account the finite absorption of the PC element were also performed. The calculations evaluated the dispersion effects of the PC layer as a function of wavelength and wave propagation direction. Dispersion is mainly affected by the physical dimensions (thickness, periodicity) of the PC layers, although absorption in the layers also plays a role. The outcome of this work was a specific PC design that, on paper at least, yielded significantly better light extraction compared to the baseline EZBright chip (with no PC) provided absorption losses could be kept to a minimum. Allowable process tolerances for the design, again on paper, were on the order of $+/-10 \%$ of the nominal critical dimensions.

\section{PC Element Materials and Process Development}

In order to improve the LED light extraction efficiency over the baseline device (no PC), optical modeling results showed that absorption in the TCO layer portion of the PC element needed to be limited to $1 \%$ or less (for the target thickness). Cree's initial TCO films exhibited significantly higher absorption and therefore much effort was directed to bring the absorption down to an acceptable level. 
Various process parameters were explored to "tune" the TCO properties. The absorption was found to be very sensitive to many different parameters. In addition, Cree found that variation of $\mathrm{TCO}$ optimization parameters can impact properties other than absorption.

Ultimately, Cree successfully developed ultra-low absorption TCO films whose other properties also met the requirements of the $\mathrm{PC} \mathrm{LED}$ design. The absorption results for all runs are compiled in Figure 5. As can be seen from the graph, steady progress was made in reducing the absorption towards the initial target of $1 \%$ and eventually surpassing it.

The improvements came about as a result of many experiments on depositing and characterizing constituent layers of the photonic crystal, including the extensive use of optical and scanning electron microscopy to look closely at the morphology of the various TCO layers.

\section{PC Element Stack}

Once the absorption of the TCO layer was at acceptable levels $(<1 \%)$, the full PC element stack was fabricated for the LED device. This PC element stack combines multiple layers for the p-contact portion of the LED device; a key metric is its reflectivity (again because the optical losses need to be very low for the PC LED to have better light extraction compared to the baseline device). Figure 6 shows the reflectivity of a PC element stack compared to a reference mirror. After a fairly extensive development effort, similar to that for the TCO, Cree was able to demonstrate PC

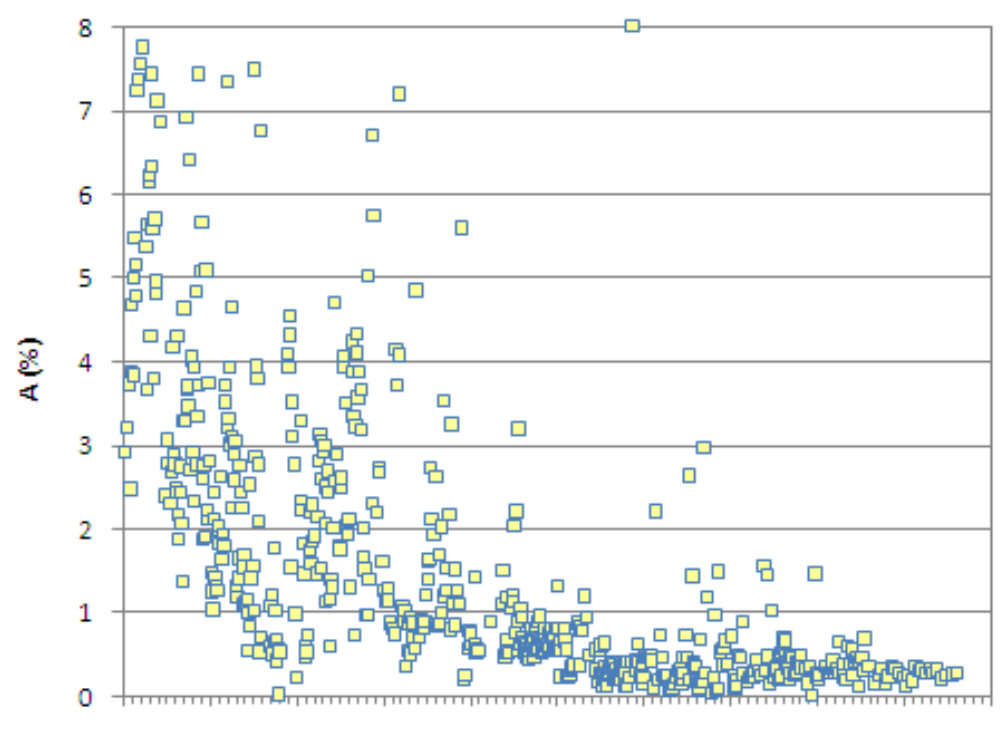

TCO Film Number

Figure 5: TCO layer absorption results as a function of time and process runs during the project. Though absorption values of $\sim 1 \%$ were realized early in the project, the reproducibility was poor. After improved PC element design and process conditions, a process with $\sim 0.5 \%$ absorption can be achieved repeatedly.

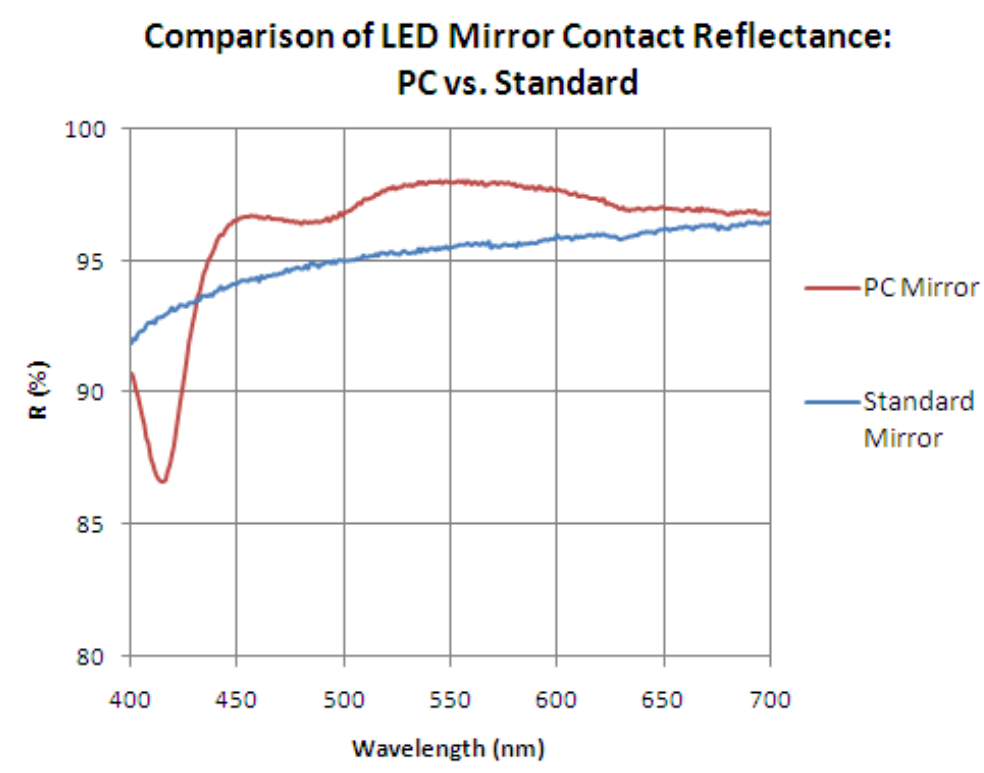

Figure 6: On-axis reflectivity as a function of wavelength for the PC stack compared to the standard baseline reflector. The on-axis reflectivity of the PC element stack exceeds baseline technology at all wavelengths of interest. 
elements whose reflectivity exceeded that of the baseline technology at all wavelengths of interest.

\section{PC LED Fabrication}

The next step was integrating the PC stack with the remainder of the LED fabrication process. The full fabrication of the device involves a number of processes, the main steps of which are:

- PC element formation

- Contact metallization

- Wafer bonding

- SiC substrate removal

A critical aspect in the fabrication of a PC-LED was maintaining the mechanical integrity of the PC stack through all the subsequent process steps involved in the LED fabrication. Initially, Cree found that the PC stack, created at the beginning of the fabrication process, was
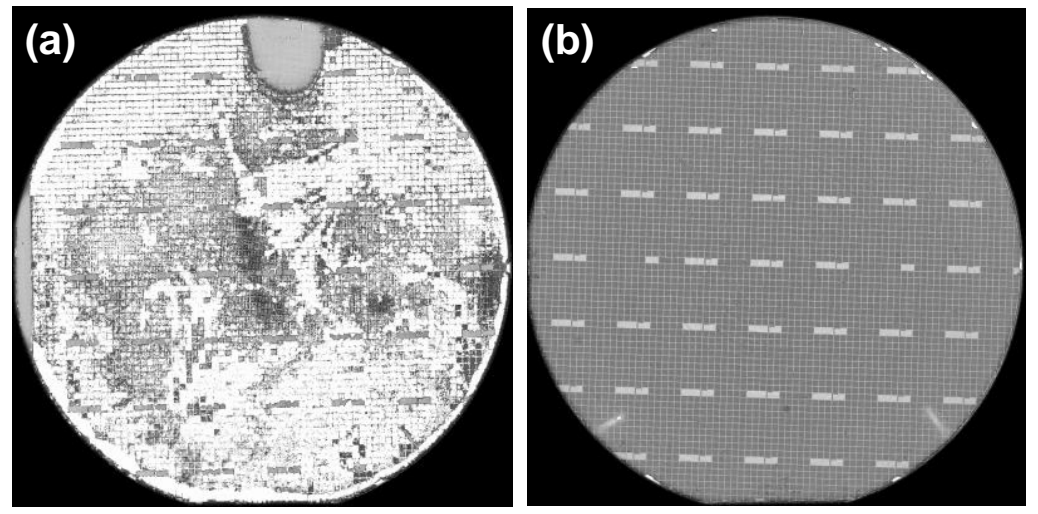

Figure 7: SAM image of bonded 3-inch PC LED wafer with (a) a poor yield and (b) a significantly increased yield as a result of process optimization. The dark areas indicate a good bond, whereas the light areas are voids. (The lighter gray rectangle areas are test devices on the wafer not poor wafer bonding.) susceptible to degradation in subsequent processing steps due to the combination of dissimilar materials in the stack and an assortment of process variables. In particular, the thermal stresses that occur during wafer bonding caused considerable problems that had to be addressed.

After PC element formation and contact metallization was completed, the LED wafer was bonded onto a carrier wafer at elevated temperatures. Scanning acoustical microscopy (SAM) was used to evaluate the quality of the wafer bond. In this technique, dark areas in the SAM image indicate a good bond, whereas light areas indicate voids or other bond failures. As seen in Figure 7a, in the early part of the project most devices showed bond failure, which was traced to mechanical failure of the PC element.

New materials used in various parts of the PC stack improved the quality of the bonding across most of the wafer. As the stack integrity was continually improved, the bonding yield across the wafer also improved. In addition to the new materials, modifications to several PC stack process parameters eventually resulted in a high quality PC stack that was able to withstand the PC-LED fabrication process steps, thus leading to very high-quality wafer bonding and final device yields (Figure 7b).

\section{PC LED Performance}

Throughout the project, once fabrication was complete, PC LED chips were packaged in Cree's XLamp test bed and their performance measured using an integrating sphere (to measure total flux and average color point) and a spectrometer (to measure the intensity as a function of wavelength). To compare LED brightness over multiple process runs, Cree uses a parameter termed "relative flux", which normalizes the measured LED flux to that of a historical Cree 
standard device and eliminates the effects of wafer-to-wafer variations. For reference, Cree's conventional EZBright LED operating at $350 \mathrm{~mA}$ has a typical relative flux value of $\sim 54$.

At the end of the first year, a PC LED was demonstrated with an average relative flux value of 54, i.e. comparable to the benchmark EZBright LED. The PC LEDs provided $501 \mathrm{~mW}$ output power at a drive current of $350 \mathrm{~mA}$. The resulting EQE was $52.2 \%$ at $350 \mathrm{~mA}$, which fell below the target EQE of 55\%. This deficit was attributed to the fact that the brighter portion of the test wafer had poor device yield and was not usable. However, the PC LED process had been shown to provide equivalent performance to the benchmark LED, an important first step. Though light output was on par with the benchmark EZBright LED, the operating voltage exceeded 4 volts, $42 \%$ percent above the desired power consumption. The median operating voltage was $4.6 \mathrm{~V}$, with some devices as low as $4.1 \mathrm{~V}$.

Table 2: Performance summary of PC LEDs during the two-year project (1 $\mathrm{mm}^{2}$ chips)

\begin{tabular}{|c|c|c|c|c|c|}
\hline PC LED & $\begin{array}{c}\text { Current } \\
(\mathrm{mA})\end{array}$ & $\begin{array}{c}\text { Power Output } \\
(\mathrm{mW})\end{array}$ & $\begin{array}{c}\text { EQE } \\
(\%)\end{array}$ & $\begin{array}{c}\text { Dom. WL } \\
(\mathrm{nm})\end{array}$ & $\begin{array}{c}\text { Operating Volt. } \\
(\mathrm{V})\end{array}$ \\
\hline Year 1 & 350 & 501 & 52.2 & 455 & 4.6 \\
\hline \multirow{2}{*}{ Year 2 } & 350 & 570 & 59.0 & 455 & 3.27 \\
\cline { 3 - 6 } & & 590 & 60.5 & 452 & 3.45 \\
\hline
\end{tabular}

Continuous improvement during the second project year led to PC LEDs with increased EQE and reduced voltage. Factors leading to this improvement include better PC designs and higher quality materials. Cree also addressed the early process repeatability issues that led to a wide performance variation between early lots due to sensitivity of the PC element fabrication steps. Additionally, fabrication processes were transferred to production tools in Cree's manufacturing site where possible, which helped eliminate some of the run-torun variation.

In brief, Cree achieved significant brightness gains and voltage improvements of PC LEDs over the second project year, as seen in Table 2. By the conclusion of the project, PC LEDs had demonstrated record performance, with some LEDs producing as much as $570 \mathrm{~mW}$ output power with low operating voltage of 3.27 $\mathrm{V}$ at a $350 \mathrm{~mA}$ drive current. The resulting EQE was 59\%.

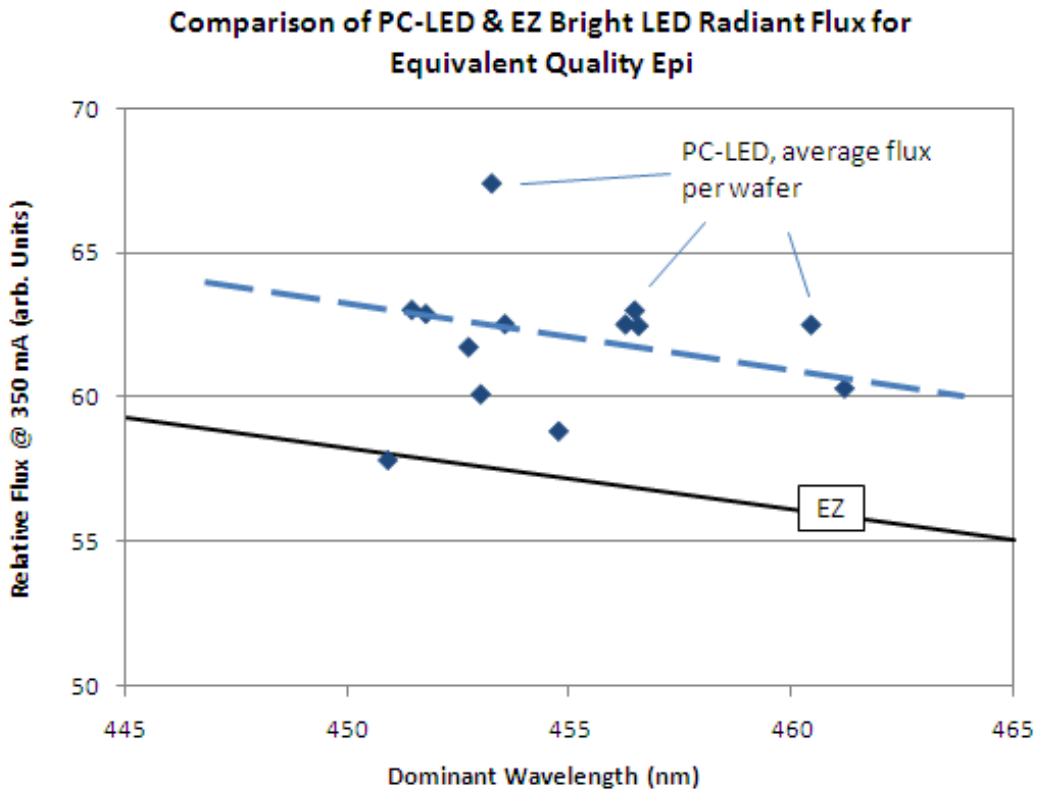

Figure 8: Relative flux as a function of wavelength for the PC LED compared to the EZBright LED production average flux value. The latest PC-LED design is $\sim 9 \%$ brighter on average than Cree's current EZBright product. 
In addition, some of the PC LEDs showed even higher EQEs above $60 \%$ although the operating voltage of those devices was slightly higher at $3.45 \mathrm{~V}$.

Compared directly, the latest PC-LED design is approximately $9 \%$ brighter on average than Cree's current EZBright product. Figure 8 shows the relative flux as a function of wavelength for the PC LED compared to the EZBright LED production average flux value. Though the EQE of the PC LEDs fell shy of the project milestone of $65 \%$, a significant improvement over the standard EZBright LED is seen. In addition, the PC LED chip architecture was carefully designed to be scaled into high volume production.

\section{PC LED Reliability}

Cree has performed preliminary reliability testing on the PC LEDs to assess if the processes and materials can provide the same performance level over the lifetime of the LED as the baseline technology.

Cree performs accelerated environmental testing using standard test methods in the LED industry to investigate the behavior of the LED under stressed testing. Our typical reliability tests run in stressed conditions (high temperature and high humidity) for 1000 hours. The LEDs are checked for drops in photometric
PC LED Preliminary Reliability: Lumens Maintenance at $350 \mathrm{~mA}, 85 \% \mathrm{RH}, 85 \mathrm{C}$

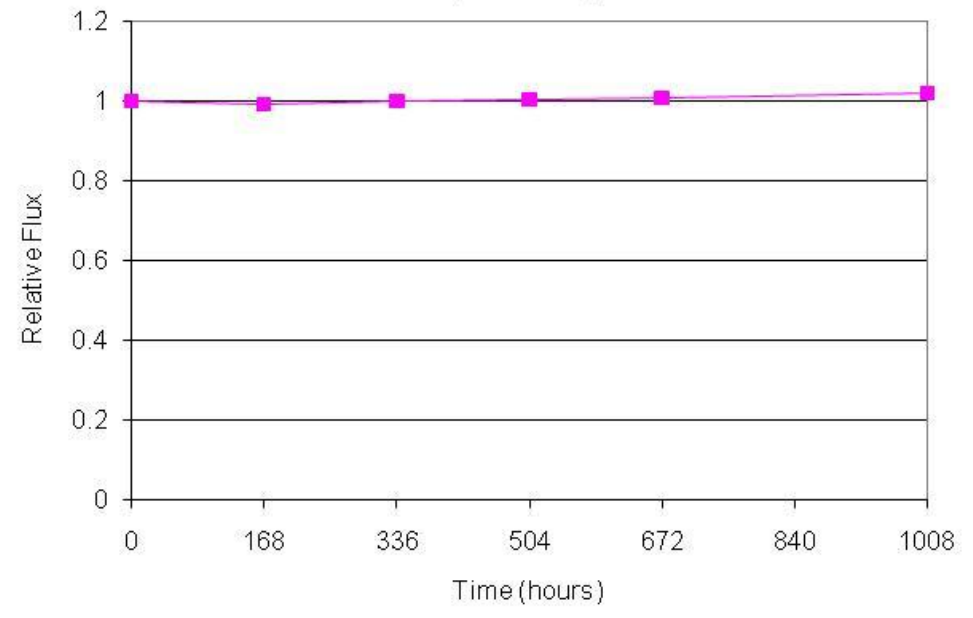

Figure 9: Normalized photometric flux observed during 1000 hours WHTOL testing for 90 blue packaged PC LED lamps tested at $350 \mathrm{~mA}$. The data corresponds to the average of all the lamps tested.

flux, changes in LED wavelength, increases in forward voltage and increases in forward leakage at various intervals throughout the 1000 hours.

Blue PC-LED lamps showed excellent performance under reliability testing, resulting in no change in flux or voltage over 1000 hours stressed operating conditions at $350 \mathrm{~mA}$. Wet high temperature operating life (WHTOL) testing results for photometric flux are shown in Figure 9, with the data corresponding to the average of all the lamps tested. In this test, 90 LEDs were packaged in Cree's standard XLamp test bed.

\section{PC LED Manufacturability}

The manufacturability of the PC element and PC stack on 3-inch diameter and larger wafers was evaluated. Cree identified critical parameters associated with the PC element and PC stack, such as layer thickness and overall stack reflectivity. Initially, the uniformity criteria were associated with critical parameters of the PC element itself, and later shifted to the LED brightness across the wafer.

Cree exceeded the Year 1 uniformity milestone of $<10 \%$ critical parameter variation across a 3inch wafer with impressive results. The PC element layer thickness and the PC stack reflectivity uniformity across a 3-inch wafer were determined using a spectrophotometer. The results are summarized in Table 3. The thickness uniformity for the PC elements was assessed 
using a notch filter test structure (the wavelength of the test structure indicates the layer thickness) resulting in a $+/-0.5 \%$ thickness variation across the 3 -inch wafer. The reflectivity of the PC stack was even better with a variation of $+/-0.4 \%$ across the 3 -inch wafer. These results were an important first step towards demonstrating the feasibility of the PC LED production processes.

Table 3: Summary of PC element and PC stack uniformity across a 3-inch LED wafer. Excellent uniformity is achieved with critical process parameters having less than a $1 \%$ variation across the wafer.

\begin{tabular}{|l|c|c|}
\hline PC LED Manufacturability & $\begin{array}{c}\text { PC Element } \\
\text { Thickness }\end{array}$ & $\begin{array}{c}\text { PC Stack } \\
\text { Reflectivity }\end{array}$ \\
\hline Uniformity across $\mathbf{3}$ " wafer & $+/-0.5 \%$ & $+/-0.4 \%$ \\
\hline
\end{tabular}

During the project's second year, the intrawafer and interwafer repeatability for fully processed PC LED wafers were evaluated in terms of LED brightness and operating voltage. Cree has demonstrated 3-inch diameter PC LED wafers with more than $90 \%$ of the devices displaying brightness within $+/-10 \%$. The normalized intensity distribution from a batch of PC LED wafers was evaluated by on-wafer probing, with $100 \%$ sampling. Figure 10 shows the normalized intensity distribution, with more than $90 \%$ of the die falling within the uniformity criteria. With regards to the small percentage of devices that were "dim", it is worth noting that even standard production LED processes have some fall out due to defects or other flaws in the fabrication steps. In terms of demonstrating the scalability of the PC LED process to large area wafers, these results effectively met the Y1.75 milestone for a 3-inch wafer process with $+/-10 \%$ brightness uniformity across the wafer. This was another important milestone towards demonstrating the basic manufacturability of PC LEDs.

Cree has also demonstrated 3-inch PC LED wafers with $80 \%$ of the devices exhibiting operating voltages of 3.0 to $3.2 \mathrm{~V}$ at $350 \mathrm{~mA}$ (note: values here are on-wafer probing; the LED voltage will increase by $\sim 200 \mathrm{mV}$ for singulated die in an XLamp package). Figure 11 shows the distribution of PC LED voltages from this batch of 3-inch

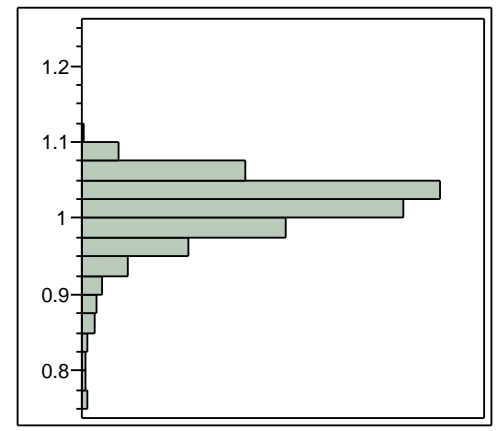

Figure 10: Normalized intensity distribution of PC LEDs on a 3inch wafer. More than $90 \%$ of the wafer falls within $+/-10 \%$ brightness.

Figure 11: Forward voltage distribution of the recent batch of 3-inch PC LED wafers showing $80 \%$ of the devices have operating voltages between 3.0 and 3.2 $V$ at a current of 20 $\mathrm{mA}$. This shows that the PC LED technology can yield a manufacturable process.

\begin{tabular}{|c|c|c|}
\hline \multicolumn{3}{|c|}{ Quantiles } \\
\hline $100.0 \%$ & maximum & 1.1072 \\
\hline $99.5 \%$ & & 1.0874 \\
\hline $97.5 \%$ & & 1.0770 \\
\hline $90.0 \%$ & & 1.0574 \\
\hline $75.0 \%$ & quartile & 1.0396 \\
\hline $50.0 \%$ & median & 1.0182 \\
\hline $25.0 \%$ & quartile & 0.9863 \\
\hline $10.0 \%$ & & 0.9463 \\
\hline $2.5 \%$ & & 0.7831 \\
\hline $0.5 \%$ & & 0.4460 \\
\hline $0.0 \%$ & minimum & 0.0952 \\
\hline \multicolumn{3}{|c|}{ Moments } \\
\hline \multicolumn{2}{|l|}{ Mean } & 1.0000395 \\
\hline \multicolumn{2}{|l|}{ Std Dev } & 0.0840035 \\
\hline \multicolumn{2}{|c|}{ Std Err Mean } & 0.0013127 \\
\hline \multicolumn{2}{|c|}{ upper $95 \%$ Mean } & 1.0026131 \\
\hline \multicolumn{2}{|c|}{ lower $95 \%$ Mean } & 0.9974659 \\
\hline \multicolumn{2}{|l|}{$\mathrm{N}$} & 4095 \\
\hline
\end{tabular}

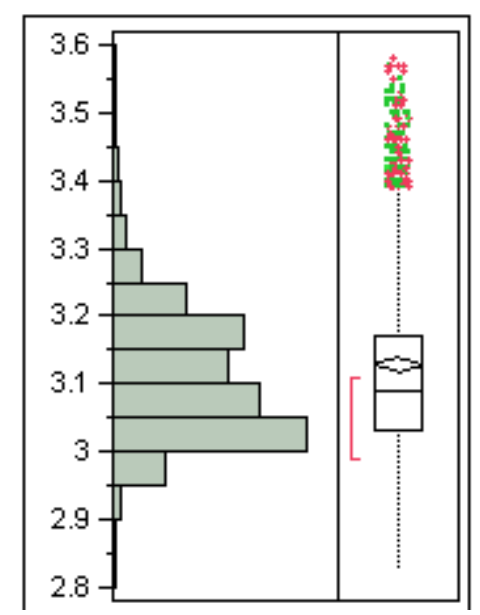


wafers. This result is further evidence that a manufacturable process can be developed based on Cree's PC LED technology.

\section{Technology Commercialization}

A product opportunity exists for commercializing the PC LEDs developed, in part, under this DOE project. PC LEDs will serve as a higher flux LED chip in the EZBright LED family. The PC LED is intended as a drop-in replacement for the current EZBright LEDs since it does not require modifications to Cree's XLamp package family based on the current chip design. Cree XLamp LEDs are the industry's highest performing power LEDs and are currently the light source for many LED lighting solutions; including Cree's award-winning LR6 and LR4 downlights and LR24 recessed LED luminaires.

The technology developed under this project will help maintain Cree's leadership in the adoption of LED lighting in general illumination. Products from a growing number of LED fixture makers utilize Cree's lighting-class LEDs to deliver real energy savings and economic benefits over a range of general illumination applications, both domestically and internationally.

\section{SECTION I - CONCLUSIONS}

During this two-year project, Cree has successfully increased the external quantum efficiency of blue LEDs to $60 \%$ - a $20 \%$ improvement over the project baseline. Specifically, blue PC LEDs have been achieved with an EQE of $60.5 \%$ at $350 \mathrm{~mA}$, yielding $590 \mathrm{~mW}$ of output power from a $1 \mathrm{~mm}^{2}$ chip. A low operating voltage of $3.27 \mathrm{~V}$ was maintained at high brightness level (570 $\mathrm{mW})$. Compared directly, the latest PC-LED design is $\sim 9 \%$ brighter on average than Cree's current EZBright LED product. Blue PC-LED lamps have solid performance under preliminary reliability testing, resulting in $100 \%$ lumen maintenance over 1000 hours operating under stressed conditions at $350 \mathrm{~mA}$.

The PC LED chip architecture was carefully designed to scale into high volume production. Cree has demonstrated a 3-inch diameter PC LED wafer with more than $90 \%$ of the devices displaying brightness within $+/-10 \%$. Cree has also demonstrated 3-inch PC LED wafers with $80 \%$ of the devices exhibiting operating voltages of 3.0 to $3.2 \mathrm{~V}$ at $350 \mathrm{~mA}$ (on-wafer probing). These results demonstrate the feasibility of production-level processes for the PC LED and hence its potential for manufacturability.

\section{SECTION II - White LED Lamp Module Design}

\section{PROBLEM BACKGROUND}

The blue photonic crystal LEDs must be combined with down-conversion phosphors in order to convert the primary LED emission to white light, as illustrated in Figure 12. While improving the blue LED chip efficiency will certainly help improve the lamp module performance, gains must also be made in the system efficiency of the module. To achieve the overall project

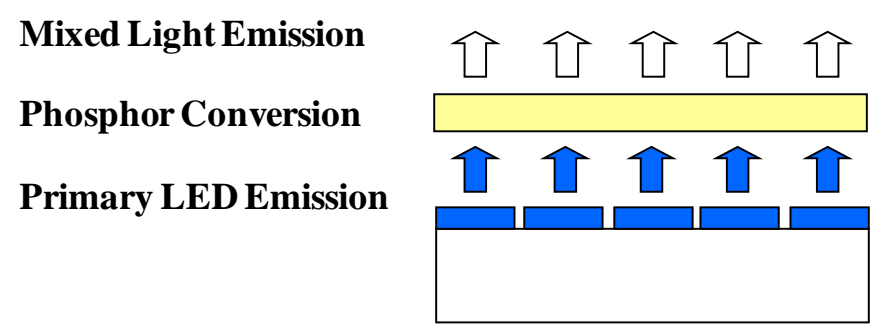

Figure 12: Schematic of the conversion of blue LED light to white light using a down-converting yellow phosphor. 
goal, Cree estimated the conversion efficiency from converting blue light to white should be approximately $68 \%$ for the white lamp module. At the start of the project, neutral white XLamp LEDs had an efficacy of 84 LPW at $350 \mathrm{~mA}$ with a corresponding in-package conversion efficiency of approximately $57 \%$. The conversion efficiency factors include the fundamental Stokes loss $(19 \%$ at $4100 \mathrm{~K})$, the phosphor IQE and re-absorption losses associated with the chip, package and phosphor scattering.

To improve down-conversion efficiency, Cree built on its existing expertise in packaged LEDs (e.g. its XLamp® product family). Better package designs and materials were developed with the intent of maximizing first-pass light output. The location of phosphor particles with respect to the chip was investigated. Additionally, Cree focused on improving the down-conversion efficiency of converting blue light to white. Up to an $81 \%$ conversion efficiency is possible excluding Stokes losses at $4100 \mathrm{~K}$.

The following efforts were undertaken in the package design:

- Reducing internal package losses due to sidewall reflection,

- Scaling to multi-chip package for high flux lamp module,

- Tailoring package geometry to (directed) emission from the PC LED.

\section{TECHNICAL PROGRESS}

\section{Reflectorless LED Module}

To improve the package efficiency, it is imperative to minimize the reflections inside the package that result in light loss. Current packages with reflectors are not ideal configurations. Increasing the package size

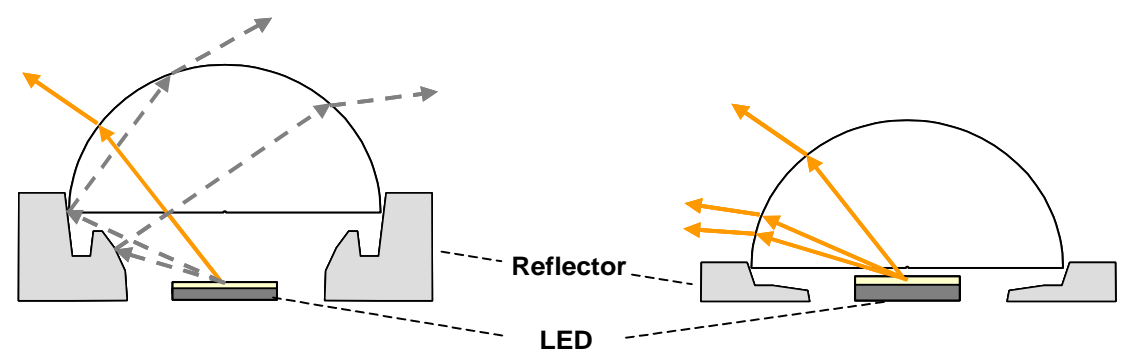

Figure 13: Schematic of a conventional chip and reflector in a lamp package (left), and a lamp package with a lowered reflector (right).

to multiple chip

configurations, necessary for the luminous output required for the final LED module, increases the potentially absorbing package area even further. One way to improve the light extraction is by removing the reflector in the assembly, as seen in Figure 13. This would increase the percentage of first-pass light significantly, without having to undergo reflections at the reflector.

Cree has demonstrated new reflectorless prototype 4-chip modules. Compared to the baseline packages, these modules have no sidewall reflector / lens retention rim but use an integrated silicone lens instead to minimize the light loss, hence improving the package efficiency. By utilizing similar materials and design rules, this work leveraged off other package development projects at

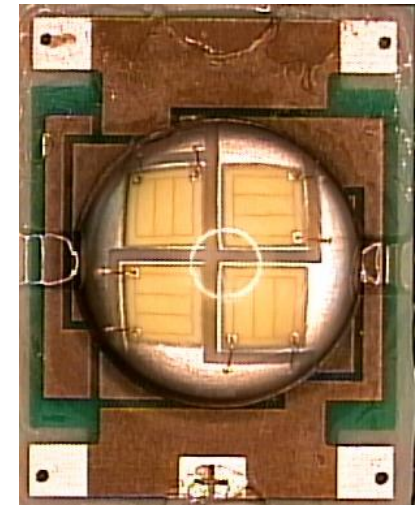

Figure 14: Photograph of the new 4-chip reflectorless prototype package $(7 \mathrm{~mm} \times 9 \mathrm{~mm})$, which satisfies the Y0.75 package design milestone. 
Cree, including a multi-color multi-chip luminaire module being developed jointly with Philips Color Kinetics on a previous DOE-supported project. A prototype 4-chip module, shown in Figure 14, satisfies the Y0.75 milestone for a package design for a generic low reflector lens.

\section{Package Conversion Efficiency}

During the first year, Cree focused on accurately quantifying optical losses in packaged LEDs using the baseline chip and phosphor materials, together with prototype packages. The experiment was an important one for the purpose of establishing an empirical and theoretical foundation on which to build. In particular, Cree was looking to separate fundamental losses (due to the down-conversion Stokes shift) from chip / phosphor / package integration losses (which, at least in principle, can be addressed). The goal of the experiment was to quantify the various optical losses associated with the phosphor and the package as a function of the fraction of down-converted light.

White LEDs were fabricated using $460 \mathrm{~nm}$ EZBright LED chips, baseline phosphor materials, and prototype packages. The color point of the 'white' LED emission was varied from a correlated color temperature (CCT) of $\sim 10,000 \mathrm{~K}$ down to $2700 \mathrm{~K}$ by changing the total amount of phosphor as well as the ratio of yellow to red phosphor. Blue LEDs (i.e. no phosphor) were also fabricated as reference devices using the same blue chips. Finished LEDs were then characterized using an integrating sphere and a spectrometer. By comparing the

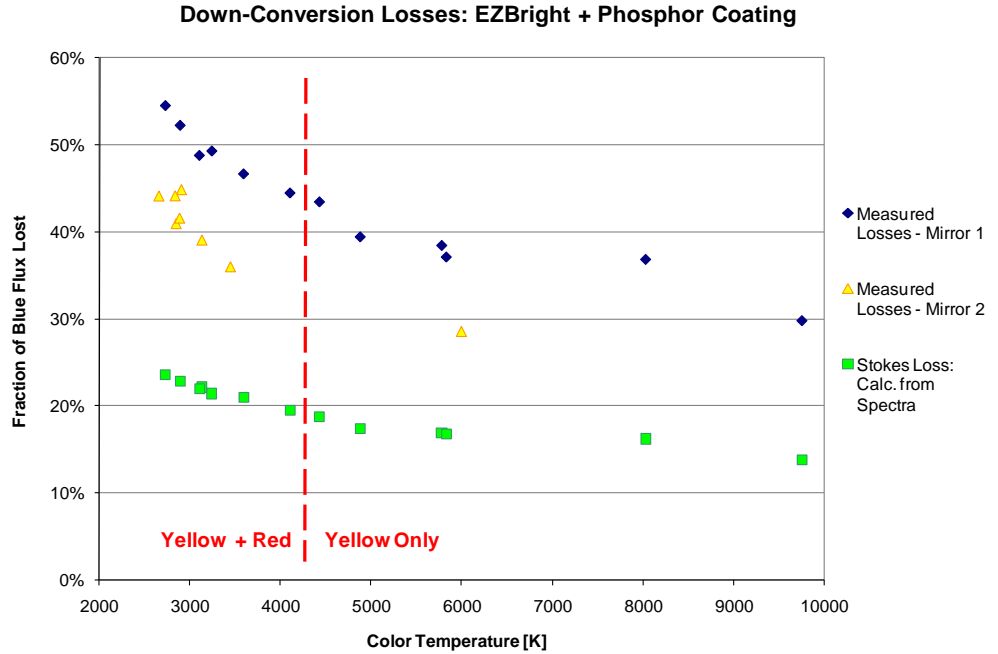

Figure 15: Baseline down-conversion losses as a function of white LED color point. Stokes loss is inherent to the down-conversion process; the total measured loss depends on absorption and scattering of photons by the chip, phosphor particles, and package. data for blue and white LEDs, Stokes loss and other optical losses were calculated for each device.

As expected, the total energy loss increases with the amount of blue light that is downconverted, reaching as much as 55\% for one set of EZBright chips, and as much as $45 \%$ for a second set of chips that included a higher reflectivity mirror contact (Figure 15). By comparison, the inherent Stokes loss reaches a maximum of only $23 \%$ (independent of the chip contact). At a color point of $4100 \mathrm{~K}$, which is the project focus, the Stokes loss is $19 \%$, while the total measured loss is $\sim 45 \%$ and $\sim 35 \%$ for the different chip sets, respectively.

\section{PC LED Integration into Package}

Cree has determined that the emission characteristics of the PC LED chips were compatible with the existing 4-chip prototype package design. Therefore, a new package design was not necessary for optimum performance of the LED component using the PC LED chip. The farfield radiation pattern of the PC LEDs in Cree's XLamp test bed was found to be the same as 


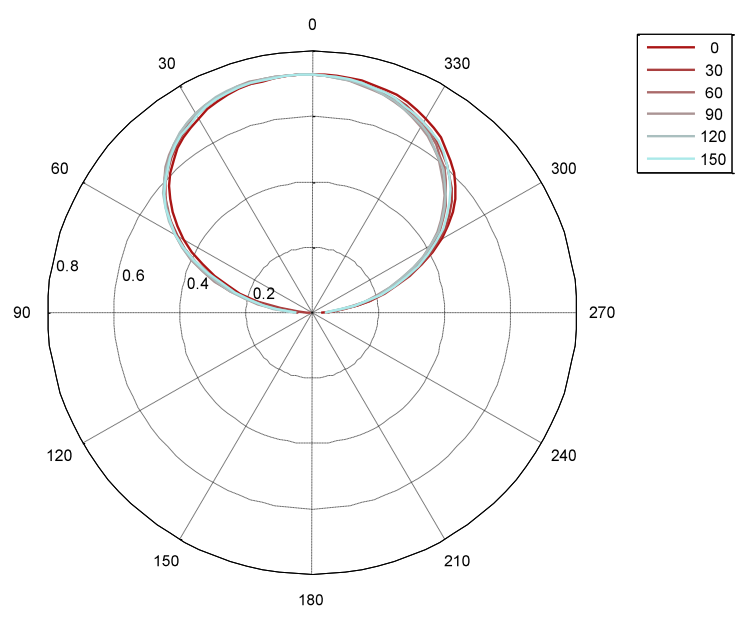

Standard LED

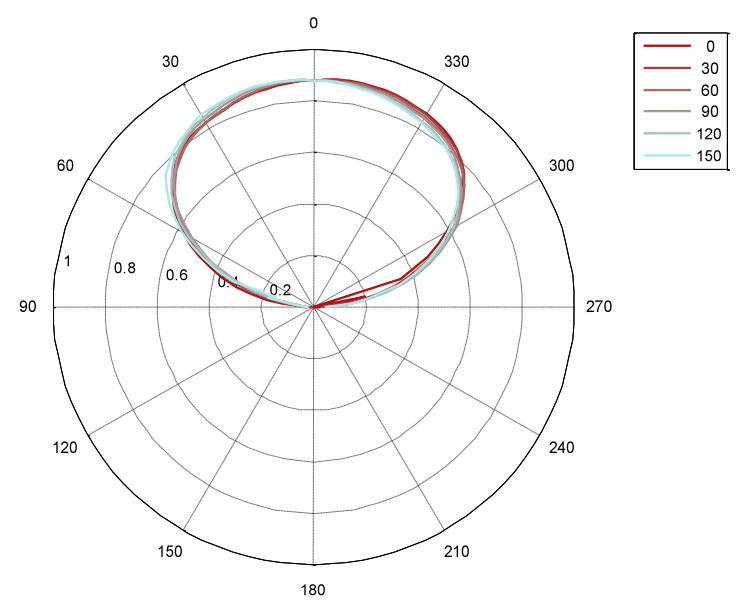

PC LED

Figure 16: The far-field radiation pattern is shown in a polar plot of intensity (a.u.) as a function of theta and phi angles for the standard LED (left) and the PC LED (right). The far-field radiation pattern of the PC LEDs in Cree's standard XLamp testbed was nominally the same as the conventional EZBright LEDs in the same package.

for conventional EZBright LEDs in the same package (Figure 16). The far-field radiation pattern was measured using a goniophotometer, which determines the spatial radiation pattern over the entire forward hemisphere of an LED. Reports in the technical literature have generally shown PC LEDs whose emission patterns have preferred directionality. Cree believes a key difference in this case lies in the specific details of our PC LED chip design, which makes it suitable as a brighter, drop-in replacement for existing chip products.

\section{White LED Lamp Module}

The project's ultimate objective was to produce a 500 lumens, 120 LPW SSL lamp module that emits at a color temperature of $4100 \mathrm{~K}$.

At the conclusion of the project's first year, Cree produced a new, prototype 4-chip module that exceeded the Year 1 efficacy milestone of 90 LPW. Cree achieved an efficacy of 91.2 LPW at a CCT of $3975 \mathrm{~K}$. This represented an $8.6 \%$ improvement over the 84 LPW laboratory result (single chip package with a reflector) at the start of the project. The total luminous flux from the module was 385 lumens at an input power of $4.2 \mathrm{~W}$, slightly below the Year 1 output flux target of 395 lumens. The performance was measured using an integrating sphere, with the results summarized in Table 4.

Table 4: Performance summary of neutral white 4-chip prototype lamp module during the twoyear project (using $1 \mathrm{~mm}^{2}$ chips).

\begin{tabular}{|c|c|c|c|c|c|c|c|c|}
\hline \multirow{2}{*}{ Lamp Module } & \multirow{2}{*}{$\begin{array}{l}\text { Current } \\
(\mathrm{mA})\end{array}$} & \multirow{2}{*}{$\begin{array}{c}\text { Flux } \\
\text { (lumens) } \\
\end{array}$} & \multirow{2}{*}{$\begin{array}{l}\text { Efficacy } \\
\text { (LPW) }\end{array}$} & \multicolumn{2}{|c|}{ Chromaticity } & \multirow{2}{*}{$\begin{array}{l}\mathrm{CCT} \\
(\mathrm{K}) \\
\end{array}$} & \multirow[t]{2}{*}{ CRI } & \multirow{2}{*}{$\begin{array}{c}\text { Input Power } \\
\text { (W) }\end{array}$} \\
\hline & & & & $x$ & y & & & \\
\hline Year 1 & 350 & 385 & 91 & 0.3809 & 0.3759 & 3975 & 78 & 4.2 \\
\hline Year 1.5 & 350 & 441 & 102 & 0.3752 & 0.3725 & 4125 & 73 & 4.2 \\
\hline Year 2 & 350 & 510 & 112 & 0.3814 & 0.3912 & 3875 & 72 & 4.5 \\
\hline
\end{tabular}


During the second project year, after further design improvements to the package and conversion efficiency, Cree built prototype 4chip modules that showed an efficacy of 102 LPW, a 12\% improvement over the 4-chip module from Year 1. This prototype module provided a total luminous flux of 441 lumens at a CCT of $4125 \mathrm{~K}$. The module's input power was $4.2 \mathrm{~W}$.

Cree continued to optimize the phosphor/chip integration scheme and phosphor material properties while keeping the PC LED chip characteristics in mind. Actual PC LEDs were inserted into the final lamp module with the conversion efficiency improvements. Ultimately, Cree demonstrated a prototype 4-chip lamp module producing 510 lumens at $3875 \mathrm{~K} \mathrm{CCT}$ with an efficacy of $112 \mathrm{LPW}\left(1 \mathrm{~mm}^{2}\right.$ chips at $\left.350 \mathrm{~mA}\right)-$ a $33 \%$ improvement over the efficacy at the start of the project. This met the overall project milestone of a 500 lumen lamp module at $4100 \mathrm{~K}$ CCT but fell short in the efficacy delivering 112 LPW instead of the target 120 LPW (Figure 17). This shortfall was attributed to the lower than targeted EQE of the blue PC LED (which was 60\% instead of the targeted 65\%).

The overall conversion efficiency at $4100 \mathrm{~K}$ improved to approximately $65 \%$ compared to $57 \%$ for baseline package at the start of the project. In addition, the single chip package performance was successfully maintained when scaling up to these 4-chip modules.

\section{Proof-of-Concept Luminaire Demonstration}

A proof-of-concept 6-inch downlight luminaire was built as part of this DOE project. The downlight produces a flux of 1700 lumens at a color point of $3842 \mathrm{~K}$ with a CRI of 94. The LM79 testing was performed on the luminaire by a CALiPER approved Photometric Test Laboratory. This luminaire met the end of the project milestone of a 1500 lumen proof-ofconcept luminaire at $\mathbf{4 1 0 0} \mathrm{K}$. Figure 18 shows a photograph of the luminaire and its illumination compared to a standard production LR6 (650 lumens). 


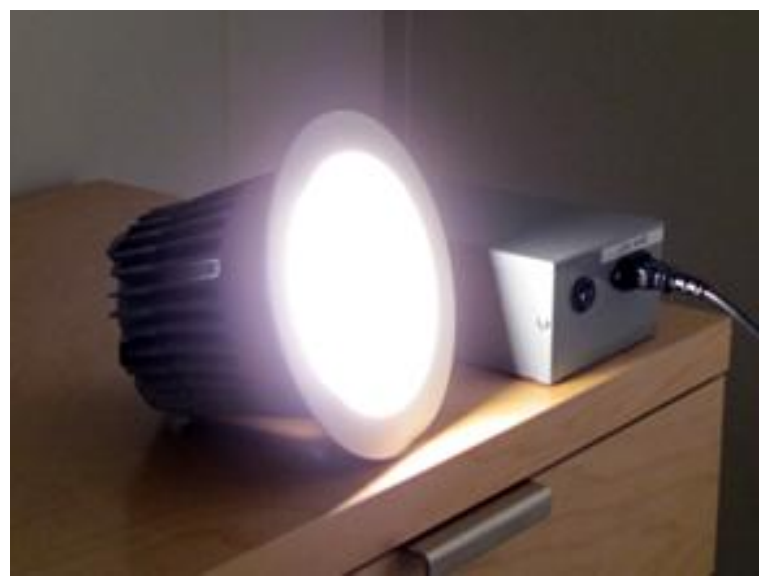

\section{Production LR6}

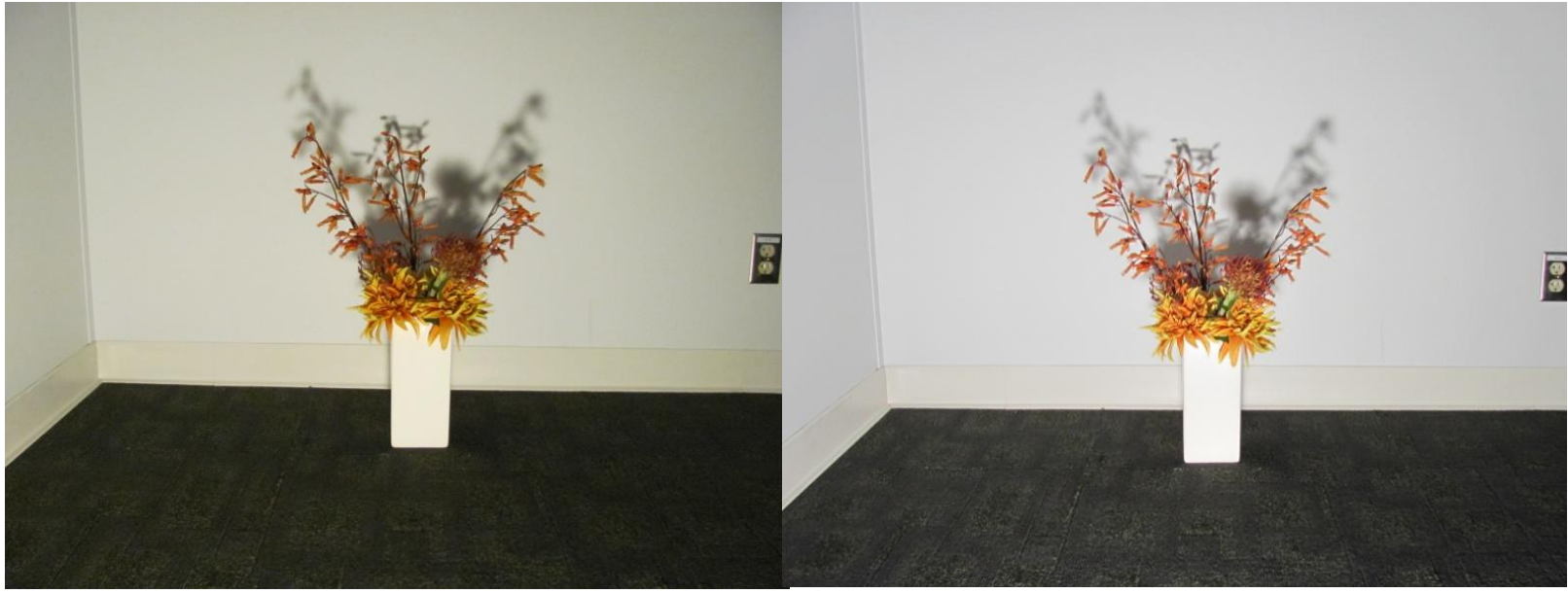

Figure 18: Photograph of prototype downlight luminaire providing 1700 lumens neutral white light at steady state complete with driver (left). As an illustration of the much higher flux of the prototype downlight compared to a production LR6 downlight (delivering 650 lumens at $2700 \mathrm{~K}$ ), the downlights were used to illuminate a vase of flowers in a dark room (below). The flux and color point of the prototype will be confirmed with third party testing by a certified laboratory.

\section{Prototype Luminaire}

\section{Technology Commercialization}

A product opportunity exists for commercializing the 4-chip prototype reflectorless packages as part of the XLamp product family. The package can utilize the EZBright chip platform including the newly developed PC LEDs. A 4-chip package can be used as the light source for many LED lighting solutions; including Cree's award-winning LED luminaires.

\section{SECTION II - CONCLUSIONS}

Cree has successfully demonstrated 4-chip neutral white LED lamp modules providing 510 lumens at a CCT of $3875 \mathrm{~K}$. This lamp module had an efficacy of $112 \mathrm{LPW}$ at $350 \mathrm{~mA}$ (using 1 $\mathrm{mm}^{2}$ chips) - a $33 \%$ improvement over the technology at project start. The overall conversion efficiency at $4100 \mathrm{~K}$ improved to approximately $65 \%$ compared to $57 \%$ for baseline package at the beginning of the project. In addition, the single chip package performance was successfully maintained when scaling up to these 4-chip modules. A proof-of-concept 6-inch downlight luminaire produced a flux of 1700 lumens at a color point of $3842 \mathrm{~K}$ and a CRI of 94. 


\section{Table 5: Summary Table of Milestone Performance}

\begin{tabular}{|c|l|c|c|}
\hline Task & \multicolumn{1}{|c|}{ Attribute } & Year 1 & Year 2 \\
\hline 2 & $\begin{array}{l}\text { PC LED Performance } \\
\left(350 \mathrm{~mA}, 1 \mathrm{~mm}^{2} \text { chips }\right)\end{array}$ & $52 \% \mathrm{EQE}$ & $\begin{array}{c}60 \% \mathrm{EQE} \\
3.23 \mathrm{~V} \text { at 350 mA }\end{array}$ \\
\hline 3 & 3-Inch Wafer Uniformity & $\begin{array}{c}\text { PC element reflectivity and } \\
\text { thickness: } \\
+/-0.4 \% \text { and }+/-0.5 \%\end{array}$ & $\begin{array}{c}\text { 90\% of PC-LEDs on 3- } \\
\text { inch wafer with +/- 10\% } \\
\text { brightness variation }\end{array}$ \\
\hline 4 & $\begin{array}{l}\text { White Module Performance } \\
\left(350 \mathrm{~mA}, 1 \mathrm{~mm}^{2} \text { chips }\right)\end{array}$ & $\begin{array}{c}385 \text { lumens, } 91 \mathrm{LPW} \\
3975 \mathrm{~K}\end{array}$ & $\begin{array}{c}510 \text { lumens, } 112 \mathrm{LPW} \\
3875 \mathrm{~K}\end{array}$ \\
\hline
\end{tabular}

